\title{
Objects parameters estimation based on optimization algorithms in active infrared thermogaphy
}

\author{
by B. Szymanik
}

\begin{abstract}
West Pomeranian University of Technology in Szczecin, Faculty of Electrical Engineering, Sikorskiego 37, 70 -
\end{abstract} 313 Szczecin, Poland

\begin{abstract}
The increasing popularity of composite materials in modern industries puts new demands on the quality control of used products. The well-established NDT methods, utilized in the metal structures evaluation, may be useful also in case of composites, but still there is as need of development of new or adaption of existing methods to composite materials properties. One of the methods is active infrared thermography (AT). In this paper the evaluation of composite samples using AT will be presented. The dedicated algorithm based on optimization methods will be used to estimate the geometrical parameters of material's defects.
\end{abstract}

\section{Introduction}

Glass fiber reinforced polymer composites become more and more popular in the all branches of modern industry. High demands on quality standards implies developing of the sophisticated and highly accurate methods of materials testing. The quality control should be possible at every stage of the material manufacturing process, as well as during the operation of finished constructions. It is well known that due to the complexity of the manufacturing process, the final composite products may contain production flaws like defects of the mould, gel coat, polymer matrix or the reinforcement. Moreover, there are defects caused by exploitation, such as delamination, humidity penetration, impact caused defects and another. Among the most common defects are impact caused holes, analyzed in this study.

The golden standard of the procedure of quality control in case of glass fiber reinforced polymer composites has not been yet developed, and there is still an open research field. The active infrared thermography, capable of fast and reliable inspection of materials, is considered one of the most promising non-destructive techniques (NDT), that may be used in case of composites [1 - 4]. The defect detection is the first stage of the quality control process. Beside the qualitative evaluation, the quantitative one - defects recognition and assessment - is very important to obtain the full information on the material under test.

In this study the dedicated numerical algorithm of solving the inverse problem for active thermography will be proposed $[5,6]$. The algorithm, based on optimization methods and finite element modelling will be used for the estimation of the geometrical (i.e. depth and size) properties of detected defects.

In our case, the inverse problem is to determine the geometrical parameters of the detected composite sample defects on the basis of the temperature distribution observable on the surface. This distribution can be obtained from the sequence of thermal images. The main assumption of our method is a strong relationship between the geometric parameters and material properties of detected defects and the temperature distribution functions. The problem solution is based on optimization algorithm, minimizing the difference between two temperature profiles - obtained numerically using finite element method, and this observable at the sample surface. Building the accurate numerical model is a crucial part of this study, and will be presented in details in a full version of this paper.

\section{Numerical modelling}

The first stage of the inverse problem solution is creating the numerical forward problem. The numerical model was prepared with COMSOL Multiphysics software and solved using Finite Elements Method (FEM). The simulated sample, with the set of defects was heated using simulated infrared heater in a form of an array of external radiation point sources.

\subsection{Model geometry and applied boundary conditions}

The numerical model of composite sample, characterized by the a low value of thermal conductivity, with a set of flaws was prepared. Holes of different dimensions (ie, depth and width) were chosen as a defect type. The model geometry and its sizes are presented in Fig. 1. Infrared heater is modelled as the array of points with given temperature and power, simulated by the external radiation source, which contributes to the incident radiative heat flux interacting with the sample with the factor proportional to the total source irradiation $G$. The interaction between the sample and radiation sources is realized by diffusive surface boundary condition, given by the formula: 


$$
q_{r}=\varepsilon\left(G-e_{b}(T)\right)
$$

where $q_{r}$-incident radiative heat flux, $\varepsilon$ - is the surface emissivity and $e_{b}(T)$ - is the blackbody hemispherical total emissive power.

In this study, the sample is heated from the back side (transmission mode), whereas the temperature is measured on the front surface, (Fig. 2). In the Table 1, all the important parameters of the model are gathered.
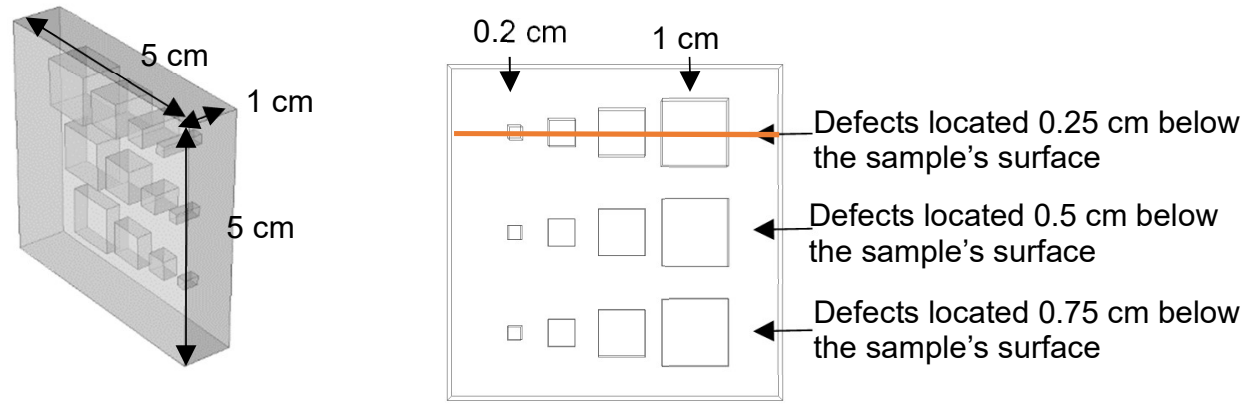

Fig. $13 D$ geometry utilized in the numerical modelling. The composite sample $(5 \times 5 \times 1 \mathrm{~cm})$ with set of rectangular holes (side lenghts: $0.2 \mathrm{~cm}, 0.4 \mathrm{~cm}, 0.7 \mathrm{~cm}$ and $1 \mathrm{~cm})$, located on three different depths $(0.25,0.5$ and 0.75 $\mathrm{cm}$ below the samples surface) was simulated
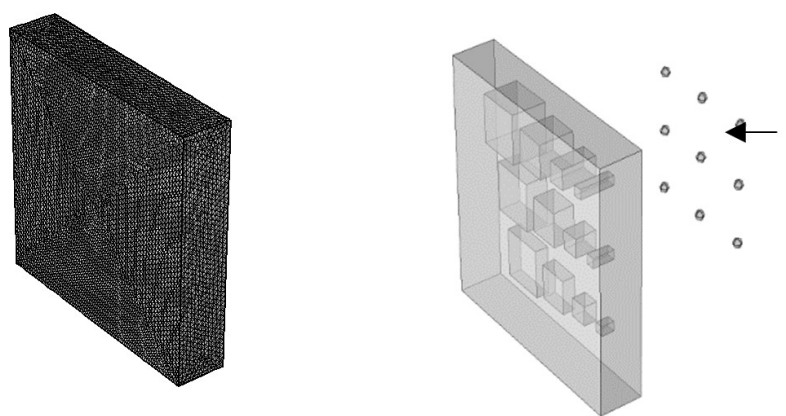

The array of external radiation points.

The back side of the sample is heated.

Fig. 2 The example of model's meshing and numerical modelling details

Table 1 Chosen model parameters

\begin{tabular}{|l|c|c|}
\hline \multicolumn{3}{|c|}{ Sample material properties } \\
\hline Density & 1420 & $\mathrm{~kg} / \mathrm{m}^{\wedge} 3$ \\
\hline Heat capacity & 1700 & $\mathrm{~J} /\left(\mathrm{kg}^{\star} \mathrm{K}\right)$ \\
\hline Thermal conductivity & 1 & $\mathrm{~W} /\left(\mathrm{kg}^{\star} \mathrm{K}\right)$ \\
\hline \multicolumn{3}{|c|}{ Heating source } \\
\hline Total Power & 900 & $\mathrm{~W}$ \\
\hline Source temperature & 1000 & $\mathrm{deg} \mathrm{C}$ \\
\hline
\end{tabular}

\subsection{The results of numerical modelling}

In the numerical modelling the total heating time was set to 30 seconds, and overall time of the simulation was 60 seconds (i.e. 30 seconds of natural cooling of the sample was included). The time step was set to 0.1 second, which gives 600 temperature distributions which may be plotted on the front side of the sample to simulate the thermovision camera measurements.

In case of inverse problem solution, the line temperature distributions are used (as a result of $2 \mathrm{D}$ problem solution). The Fig. 3 presents exemplary temperature distribution plotted on the front side of the simulated composite sample and the linear temperature distributions plotted along the line indicated in Fig. 1 (crossing the set of simulated defects). It can be noticed that the linear temperature distributions show the dependency of the obtained curves on the defects sizes and depths. This strong dependency is used later in the inverse problem solution. 

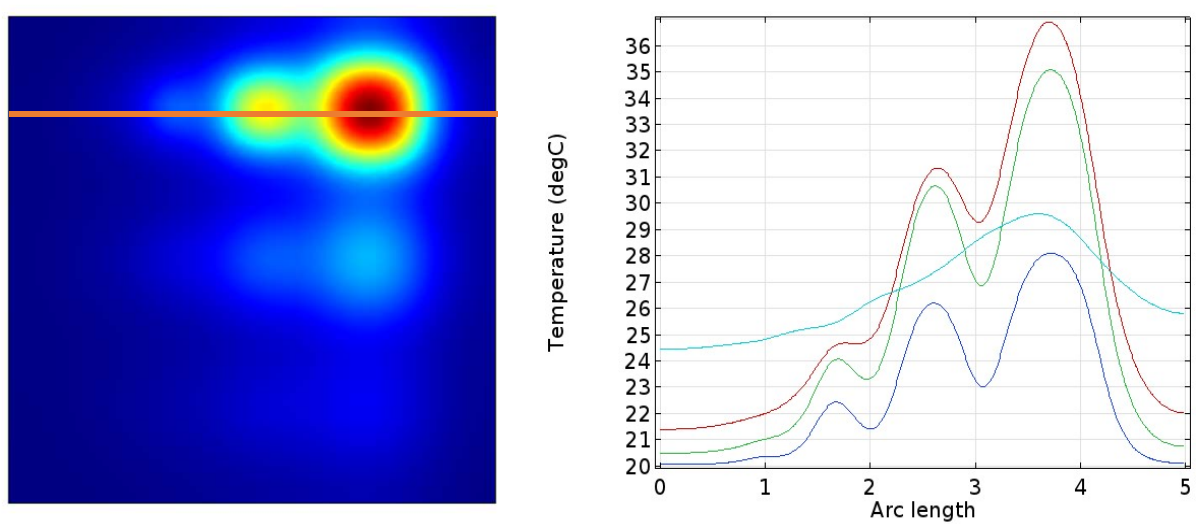

Fig 3 Left: Temperature distribution observed at the front side of modelled sample after 30 seconds of simulation time. Right: the linear temperature distributions for different time steps plotted along the indicated line - blue line - after 10 seconds of heating, green line - after 20 seconds of heating, red line - after 30 seconds of heating, aquamarine line - after 60 seconds of simulation

\section{Inverse problem solution}

The method of inverse problem solution presented here bases on the optimization algorithm. In this case the multiple solving of the forward problem is demanded. The 3D FEM models generally take much more time to be solved, than the 2D ones. Therefore, in this study the 2D model, understood as a cut plane of a 3D model (Fig. 4), was taken into account. This way it is easy to evolve this problem to 3D one, by analysing the multiple cut planes.

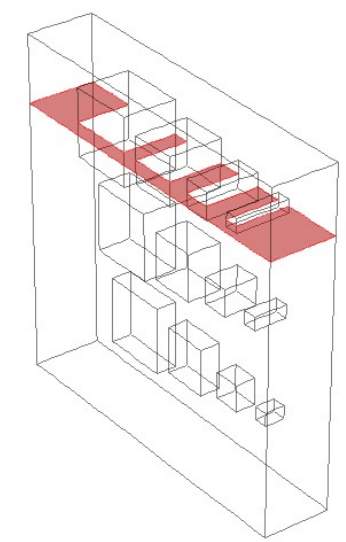

Fig. 4 The analysed 2D problem, understood as the cut plane of the $3 D$ model

The optimized geometrical parameters were defect's width and depth, as it is indicated in the Fig. 5.

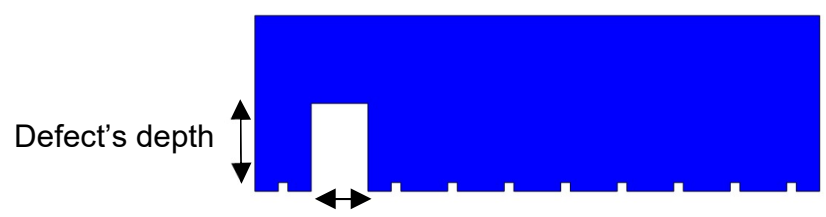

Defect's width

Fig. 5 The optimization parameters shown for one exemplary defect

The total number of possible defects is set to 10 , which means, that the defects number was also given as a non-direct optimisation parameter. The optimization input vector has 20 coordinates (i.e each defect width and depth), the search space was set to fit the range from "no defect" (the width and depth of each defect is set to $0.001 \mathrm{~m}$ ), to "no sample" (the width of each defect is set to $0.0059 \mathrm{~m}$ and depth to $0.019 \mathrm{~m}$ ), which is shown in Fig. 6 . 

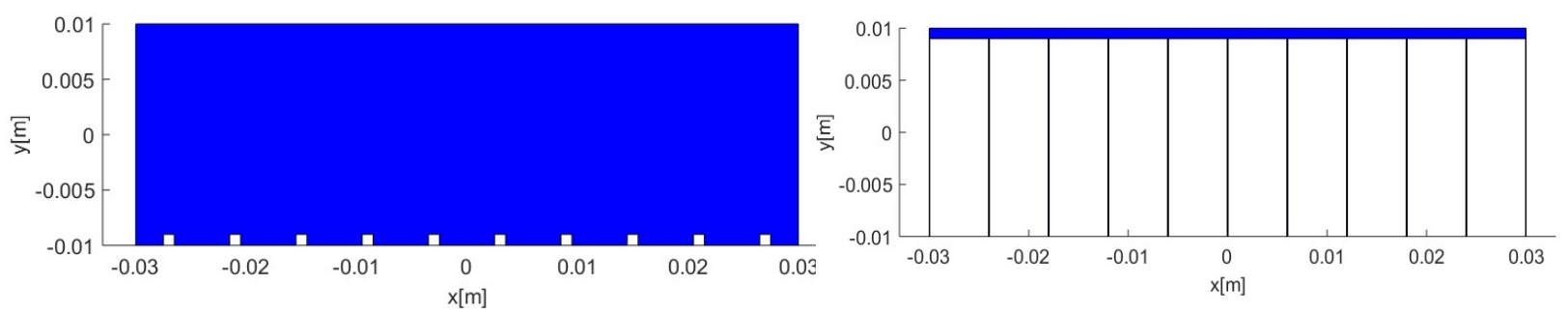

Fig. 6 The optimisation algorithm search space. Left: "no defect" case, right: "no sample" case

The inverse problem solution flaw chart is presented in Fig. 7.The pattern search algorithm was used. The problem was solved using Matlab with Comsol software: the forward problem for each step was solved in Comsol, the optimisation was performed using Matlab optimization toolbox. In each step the linear temperature distribution for the given input vector of defects parameters was computed and compared with the goal distribution. The optimisation goal was to minimise the difference between the goal $\left(T_{\mathrm{opt}}\right)$ and obtained distribution $(T)$, defined by MSE:

$$
F=\sum_{i=1}^{60} \sqrt{\left(T_{\text {opt }}(i)^{2}-T(i)^{2}\right)}
$$

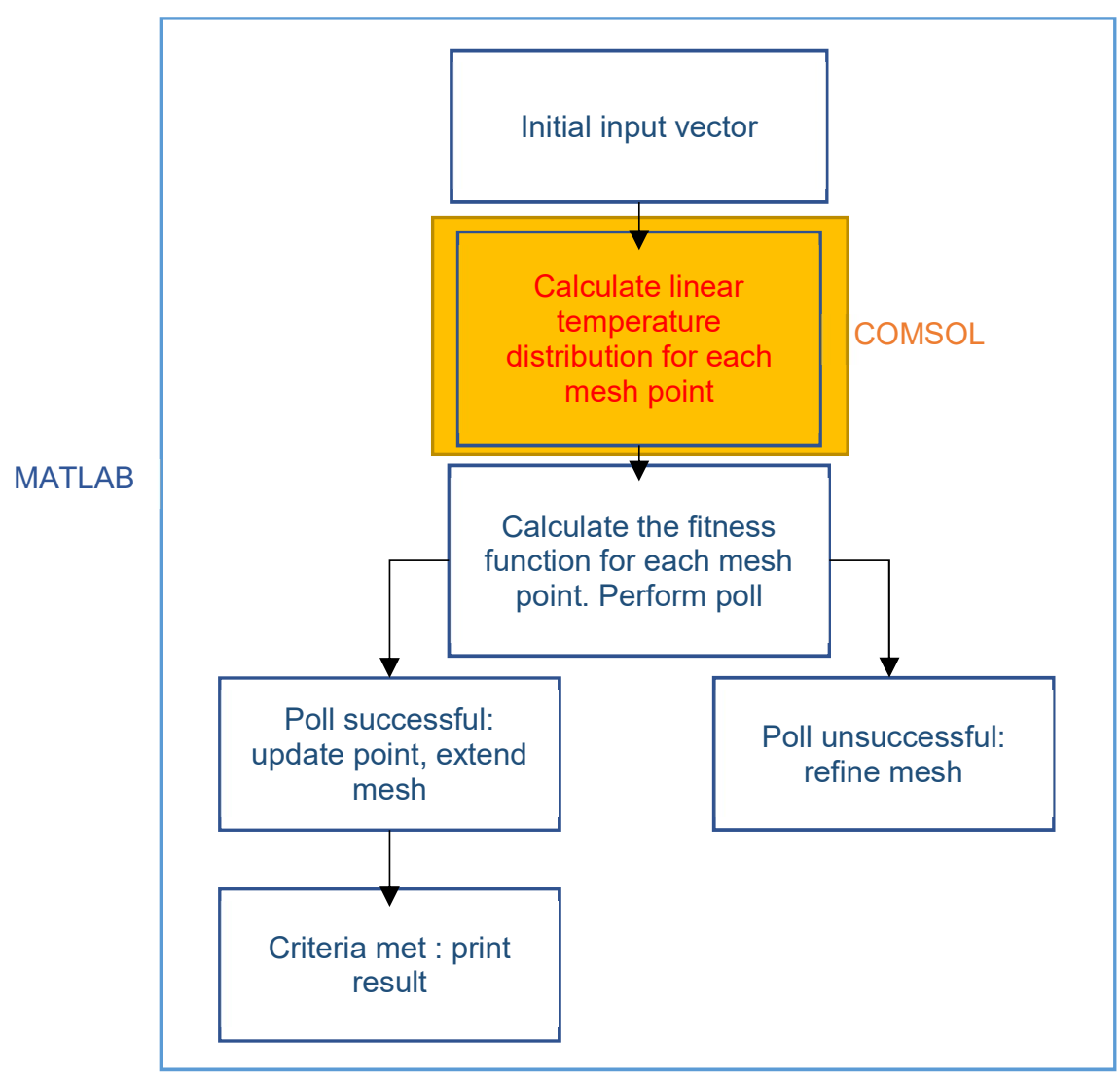

Fig. 7 Inverse problem solution flow chart

\subsection{Inverse problem solution results}

The sample with three defects of width $0.005 \mathrm{~m}$ and depth equal to $0.015 \mathrm{~m}$ was chosen to test the inverse problem solution algorithm. The defects 3, 5 and 7 were chosen to be "active", which means that the other defects were set to "no defect" mode (their sizes were set to value $0.001 \mathrm{~m}$ ). The goal geometry and the temperature distribution obtained after 30 seconds of heating is shown in Fig. 7 

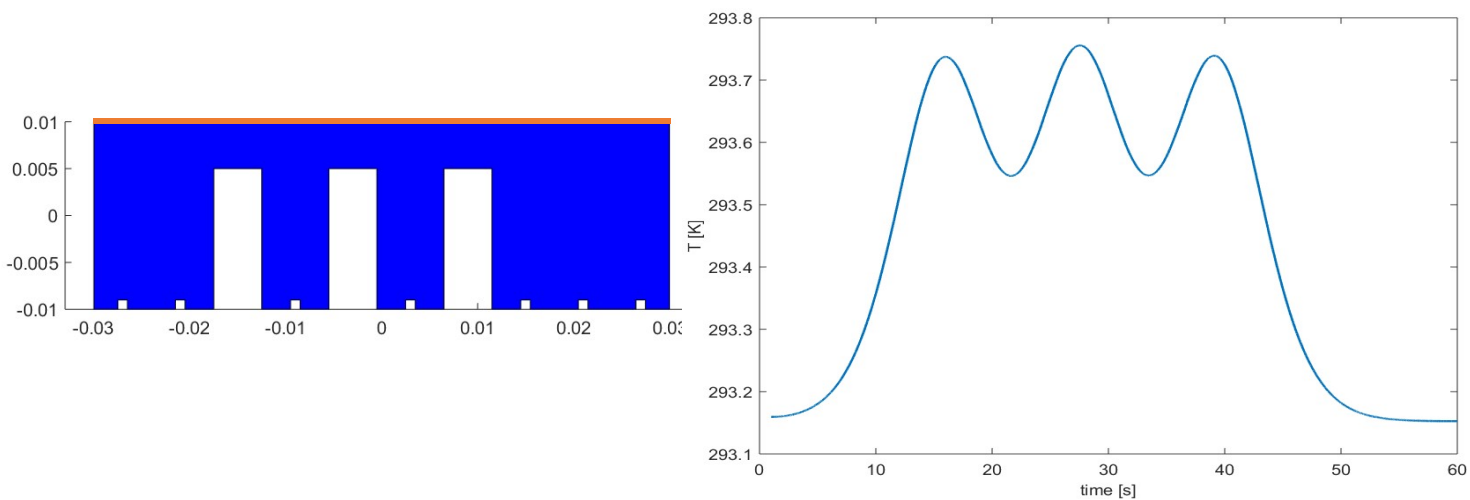

Fig. 7 The optimization goal: left: optimum geometry, right: the optimum temperature distribution obtained for 30 seconds of simulation along the indicated line

The optimization was performed in 150 iterations, and reconstructed object is presented in the Fig. 8. It can be seen that defect 5 was reconstructed, whereas the defects 3 and 7 were substituted by two neighbourhood defects of different depths. The reconstructed defects 2 and 8 have $0.001 \mathrm{~m}$ of width, therefore should be considered as "no defect" mode.

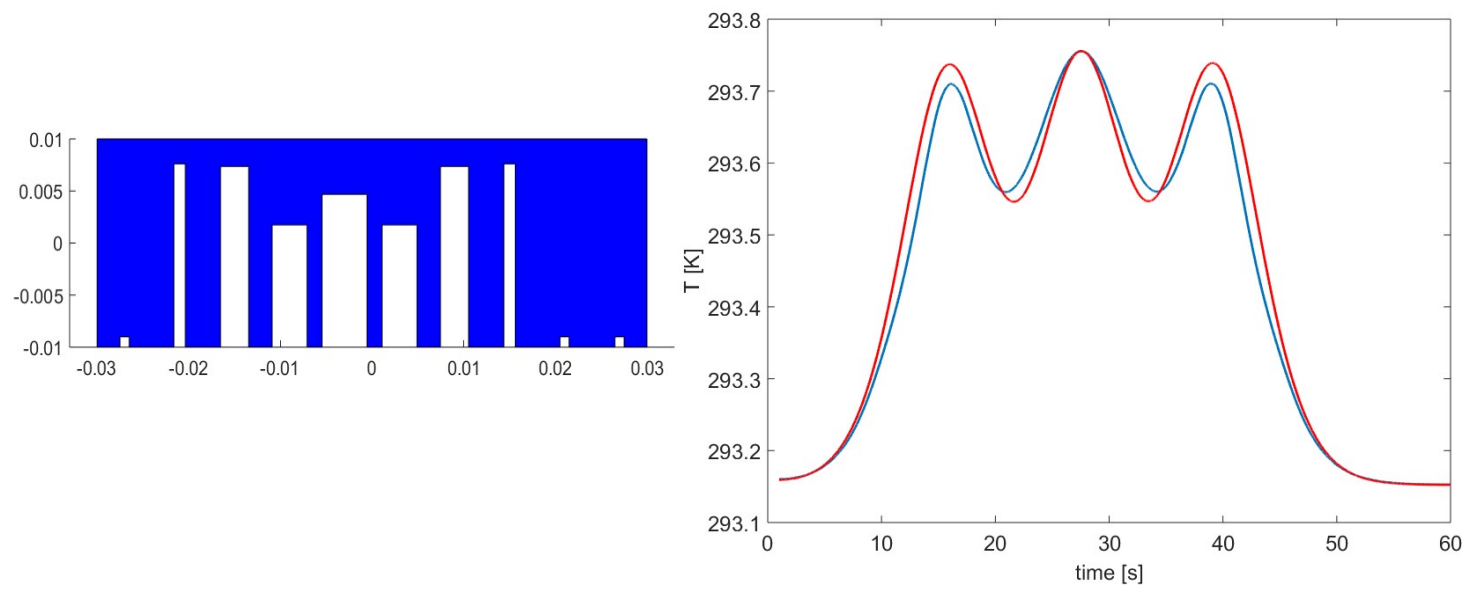

Fig. 8 The shape reconstruction results. Right: obtained geometry, left: the comparison between the goal (blue) temperature distribution and obtained (red) one

\section{Conclusion}

In this article the concept of the defects parameters estimation basing on the temperature distribution was discussed. The inverse problem solution using the pattern search optimization algorithm and finite element modelling was used for the estimation of the geometrical (i.e. depth and size) properties of detected defects. It was shown that the geometry reconstruction provides some level of accuracy, but more effort should be put on the optimization assumptions to get the better results. The next step of the research will be 3D geometry reconstruction on the basis of multiple cut planes.

\section{REFERENCES}

[1] X. P. V. Maldague, T. S. Jones, H. Kaplan, S. Marinetti and M. Prystay "Chapter 2: Fundamentals of Infrared and Thermal Testing: Part 1. Principles of Infrared and Thermal Testing," in Nondestructive Handbook, Infrared and Thermal Testing, Volume 3, X. Maldague technical ed., P. O. Moore ed., 3rd edition, Columbus, Ohio, ASNT Press, 2001, 718 p.

[2] K.K. Ghosh, V.M. Karbhari, "A critical review of infrared thermography as a method for non-destructive evaluation of FRP rehabilitated structures", Int. J. Mater. Prod. Technol., 25 (2006), pp. 241-266

[3] Bin Yang, Lixin Zhang, Weidong Zhang, Yibo Ai, "Non-destructive testing of wind turbine blades using an infrared thermography: A review", Materials for Renewable Energy and Environment (ICMREE) 2013 International Conference on, vol. 1, pp. 407-410, 2013.

[4] Ruizhen Yang, Yunze He, "Optically and Non-optically Excited Thermography for Composites: A Review", Infrared Physics \& Technology, pp. , 2016, ISSN 13504495. 
[5] Szymanik, B. (2014), "Inverse problem solution in landmines detection based on active thermography", Radioengineering , Vol. 23 No. 4, pp. 1203-1207

[6] Szymanik B., Wołoszyn M.: "Magnetic and infrared thermography methods in detection of antipersonnel landmines". Compel - International Journal for Computation and Mathematics in Electrical and Electronic Engineering. Vol. 35, Iss. 4 (2016), p. 1323-1337 